\title{
Automated SNOMED CT concept and attribute relationship detection through a web-based implementation of cTAKES
}

\author{
Martijn G. Kersloot ${ }^{1 *} \mathbb{D}$, Francis Lau ${ }^{2}$, Ameen Abu-Hanna', Derk L. Arts ${ }^{1}$ and Ronald Cornet ${ }^{1}$
}

\begin{abstract}
Background: Information in Electronic Health Records is largely stored as unstructured free text. Natural language processing (NLP), or Medical Language Processing (MLP) in medicine, aims at extracting structured information from free text, and is less expensive and time-consuming than manual extraction. However, most algorithms in MLP are institution-specific or address only one clinical need, and thus cannot be broadly applied. In addition, most MLP systems do not detect concepts in misspelled text and cannot detect attribute relationships between concepts. The objective of this study was to develop and evaluate an MLP application that includes generic algorithms for the detection of (misspelled) concepts and of attribute relationships between them.

Methods: An implementation of the MLP system CTAKES, called DIRECT, was developed with generic SNOMED CT concept filter, concept relationship detection, and attribute relationship detection algorithms and a custom dictionary. Four implementations of cTAKES were evaluated by comparing 98 manually annotated oncology charts with the output of DIRECT. The $\mathrm{F}_{1}$-score was determined for named-entity recognition and attribute relationship detection for the concepts 'lung cancer', 'non-small cell lung cancer', and 'recurrence'. The performance of the four implementations was compared with a two-tailed permutation test.
\end{abstract}

Results: DIRECT detected lung cancer and non-small cell lung cancer concepts with $\mathrm{F}_{1}$-scores between 0.828 and 0.947 and between 0.862 and 0.933 , respectively. The concept recurrence was detected with a significantly higher $\mathrm{F}_{1}$-score of 0.921 , compared to the other implementations, and the relationship between recurrence and lung cancer with an $\mathrm{F}_{1}$-score of 0.857 . The precision of the detection of lung cancer, non-small cell lung cancer, and recurrence concepts were $1.000,0.966$, and 0.879 , compared to precisions of $0.943,0.967$, and 0.000 in the original implementation, respectively.

Conclusion: DIRECT can detect oncology concepts and attribute relationships with high precision and can detect recurrence with significant increase in $\mathrm{F}_{1}$-score, compared to the original implementation of cTAKES, due to the usage of a custom dictionary and a generic concept relationship detection algorithm. These concepts and relationships can be used to encode clinical narratives, and can thus substantially reduce manual chart abstraction efforts, saving time for clinicians and researchers.

Keywords: Chart abstraction, Natural language processing, Electronic health records, Algorithms, SNOMED CT

\footnotetext{
* Correspondence: m.g.kersloot@amsterdamumc.nl

${ }^{1}$ Department of Medical Informatics, Amsterdam Public Health Research

Institute, Amsterdam UMC, University of Amsterdam, Meibergdreef 9, 1105AZ

Amsterdam, The Netherlands

Full list of author information is available at the end of the article
}

(c) The Author(s). 2019 Open Access This article is distributed under the terms of the Creative Commons Attribution 4.0 International License (http://creativecommons.org/licenses/by/4.0/), which permits unrestricted use, distribution, and reproduction in any medium, provided you give appropriate credit to the original author(s) and the source, provide a link to the Creative Commons license, and indicate if changes were made. The Creative Commons Public Domain Dedication waiver (http://creativecommons.org/publicdomain/zero/1.0/) applies to the data made available in this article, unless otherwise stated. 


\section{Background}

Much of the data present in Electronic Health Records (EHRs) are stored as unstructured free text [1] as clinicians often resort to making free-text notes, despite available coding options [2]. The use of free text should be taken into account when EHR data are reused for other purposes [3], since data reuse for research and development of clinical decision support tools can improve healthcare [4]. However, using freetext notes for searching, summarizing, statistical analysis, and as input for decision support systems is challenging [5].

One of the tasks of natural language processing (NLP) methods, named-entity recognition, aims to extract structured information from free text that is less expensive and time-consuming than extracting it manually [6]. NLP in the medical field, medical language processing (MLP), is more challenging than NLP in various other fields since clinical texts have different grammar, contain ambiguous abbreviations (i.e., the same set of letters has multiple meanings), and contain more misspellings [1, 7]. Recent studies show that MLP can successfully be used for several purposes including deriving comorbidities from the EHR [8], detecting adverse events [9], and finding eligible patients for clinical trials by attaching clinical concepts to patient charts (encoding) [10]. Furthermore, MLP has been proven successful in extracting diagnoses from free-text notes from the EHR, thereby reducing manual chart abstraction efforts. It can, for example, be used to automatically detect the recurrence of breast cancer in patient charts, reducing the number of manually reviewed charts by $90 \%$ [11]. Other research shows that MLP can identify uncodified diabetes cases, leading to a more complete ascertainment of diagnoses and, thus, better information provision and targeted care for patients [12].

MLP systems include multiple algorithms to process free text and extract information from it. Clinical Text
Analysis and Knowledge Extraction System (cTAKES) is an open-source MLP system from The Apache Software Foundation [13]. It is based on the Unstructured Information Management Architecture (UIMA) framework and the OpenNLP toolkit [13]. cTAKES provides linguistic and semantic annotations for unstructured free text [13] using SNOMED CT [14] and RxNorm [15] dictionaries. cTAKES is designed to be modular and extensible at the information model and method levels, ensuring that it is suitable for a variety of use cases [16].

MLP algorithms have been implemented in various systems. A recent systematic review has shown that most implementations of MLP algorithms are institution-specific, address only one clinical need, might be overfitted, and thus not scalable [17]. In addition, most MLP systems do not detect concepts in misspelled text, e.g. 'Smll cell lng cancer', only detect unqualified relationships (e.g. Non-small cell lung cancer relates in a way to Recurrent, Fig. 1.1) between concepts or their instances, and cannot detect attribute relationships, e.g. Non-small cell lung cancer with Recurrent as Clinical course (Fig. 1.2). Attribute relationships make the type of relationship between concepts or their instances explicit (e.g. Clinical Course in Fig. 1.2).

Since most MLP systems do not offer these algorithms, this study aimed to develop a cTAKES implementation that includes generic algorithms for the detection of concepts from properly spelled and misspelled descriptions and attribute relationships between these concepts. The implementation is evaluated by encoding free-text oncology charts to detect charts that describe recurrent non-small cell lung cancer, and use these outcomes to calculate the $\mathrm{F}_{1}$-score.

\section{Material and methods cTAKES}

cTAKES enables encoding through several algorithms, which are included in several pipelines. We used cTAKES' AggregatePlaintextFastUMLSProcessor pipeline (e.g. the

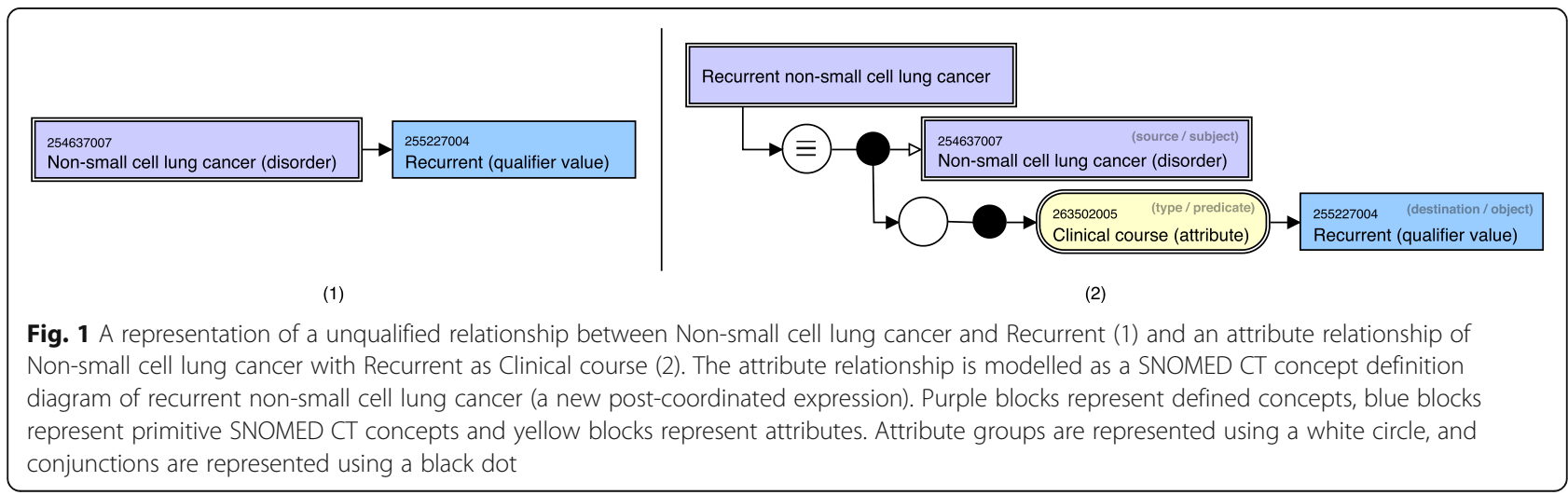


output of one algorithm becomes the input to the next [18]), as shown in Fig. 2, for the pre-processing and processing of free-text clinical narratives, since it uses the Unified Medical Language System (UMLS) [19] as its dictionary. In this project, we focus on the SNOMED CT concepts that are included in the UMLS, as the hierarchical and relational structure of SNOMED $\mathrm{CT}$ allows us to determine and define relationships between medical concepts.

\section{Development of an MLP tool}

Our project involved the development of a cTAKES implementation named Disease Information and Relationship ExtraCtion Tool (DIRECT). cTAKES provides a generic way of concept matching (through dictionary look-up), and detection of syntactic relationships and temporal references. However, it does not detect attribute relationships between concepts (e.g. Small cell carcinoma of lung with Recurrent as Clinical course) and does not match concepts in misspelled text. DIRECT was designed to filter SNOMED CT concepts matched by cTAKES, detect concepts that cTAKES did not detect, and detect attribute relationships between the detected concepts (example shown in Fig. 1.2). Figure 3 shows the workflow of DIRECT. When a user uploads a document or enters free text in the web interface of the tool (Fig. 3.1), DIRECT calls the cTAKES Application Programming Interface (API, Fig. 3.2) to obtain annotations from cTAKES. The detected annotations are processed in DIRECT (Fig. 3.3) and the result is returned to the user (Fig. 3.4).

\section{cTAKES API}

An API was developed to communicate with cTAKES. It sends text or the contents of a file, received from an HTTP POST request, to the pipeline. The API sets up a UIMA Java environment with cTAKES and the received text and runs the AggregatePlaintextFastUMLSProcessor pipeline. After the pipeline has annotated the text, the results are parsed and returned as an XML file by the API, which is used by the processing algorithm of DIRECT. The XML file contains the detected syntactic relationships and concept identifiers of UMLS concepts, accompanied with the spans of the related terms in the text.

\section{DIRECT}

DIRECT is a general-purpose web application that allows users to annotate free text originating from a clinical source by sending it to cTAKES using the cTAKES API. Users can focus on the presence of specific concepts (e.g. Primary malignant neoplasm of lung) and their children (e.g. Small cell carcinoma of lung is a Primary malignant neoplasm of lung) or the relationship between concepts, since DIRECT's algorithms are generic and do not specifically focus on specific concepts. After the text is processed by cTAKES, DIRECT uses algorithms to detect and filter SNOMED CT concepts, detect relationships between concepts, and uses those relationships to detect attribute relationships. These processing algorithms are described below (SNOMED CT concept filter, Concept relationship detection, and Attribute relationship detection). The output of the annotation and the algorithms is formatted and shown to the user.

SNOMED CT concept filter The SNOMED CT concept filter algorithm extracts the most relevant concepts from the output of cTAKES. It derives the SNOMED CT concepts related to the UMLS concept identifier provided by cTAKES and checks the status of every concept (e.g. active if the concept is still in use in

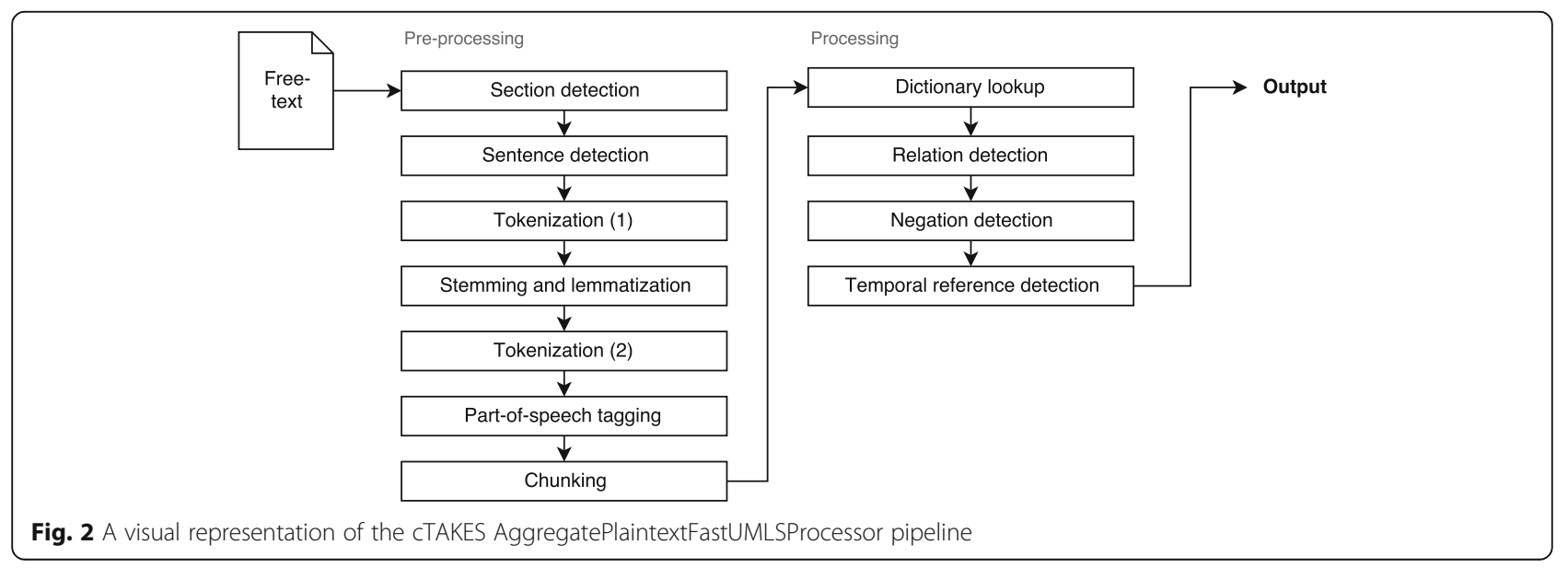




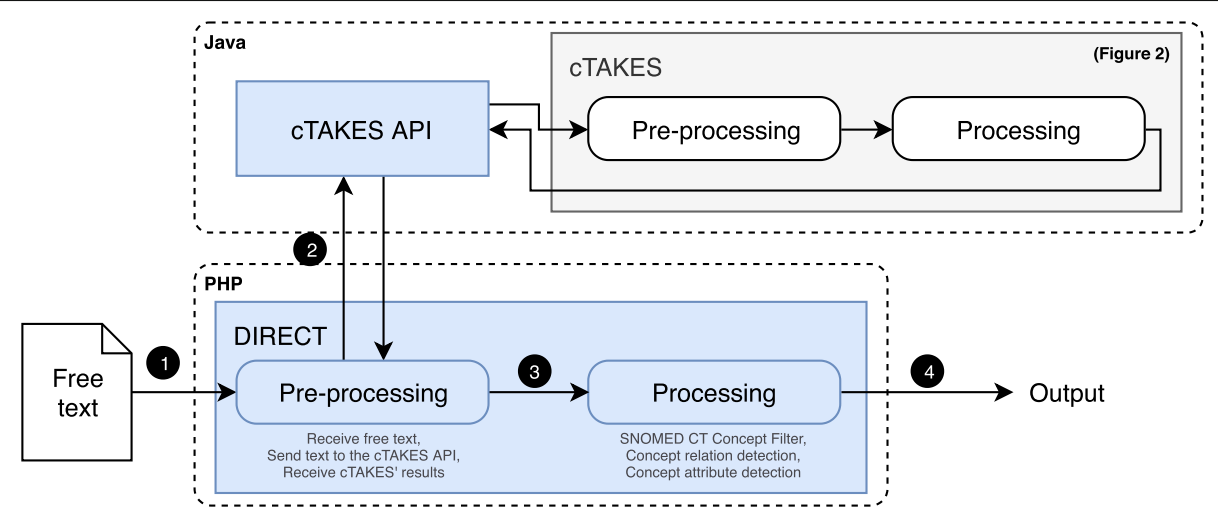

Fig. 3 A visual representation of DIRECT in relation to CTAKES. API: Application programming interface. Blue blocks represent developed components, rounded blocks MLP algorithms

SNOMED CT, inactive if not). If the concept is inactive, the filter derives its substitute concepts. Once only active concepts are selected, the algorithm detects if the term listing the concept overlaps with other terms, thus determining if multiple concepts are detected in the same span. In case of an overlap, the algorithm selects the concept associated with the longest term (e.g. 'Lung cancer': instead of 'Lung'). Finally, the algorithm analyzes every span in the text to filter duplicate concepts and to select the most detailed concept in case of a parent-child relation.

Concept relationship detection This algorithm uses cTAKES' ConllDependencyNode identifier to obtain syntactic relationships between words (tokens) that are detected in the text (e.g. in 'recurrent cancer', 'recurrent' is an adjective that relates to the noun 'cancer', Fig. 4.1). The concept relationship detection algorithm matches words from the syntactic relationships (dependencies), to concepts that are detected in the same span. First, the algorithm includes words that have nominal subject (nsubj), adjectival modifier (amod), modifier of nominal (nmod), adverbial modifier (advmod), noun compound modifier (nn), attribute (attr), direct object (dobj), object of a preposition (pobj) and modifier in hyphenation (hmod) relationships (Fig. 4.1) [20]. Next, the algorithm detects if there are concepts detected by cTAKES in the span that the word is part of ('recurrent' in Fig. 4.2). The relationship detection algorithm also tries to match words to SNOMED CT concepts, in case the concept was not detected by the cTAKES NER algorithm, using the same syntactic relationships. It includes free-text descriptions that partly match a SNOMED CT description, calculated by a similarity detection algorithm [21]. Alternative spellings as 'nonsmall cell lung cancer' and 'non small cell lung cancer' will now map to the description in SNOMED: 'non-small cell lung cancer'. This similarity detection algorithm is based on PHP's built-in similar_text function [22]. The algorithm calculates the percentage of similarity by dividing the result of matching characters in two descriptions by the average of the descriptions' lengths. We included descriptions that matched a concept description for at least 95\%, corresponding with a 5\% error margin. This error margin did not result in false positive outcomes. Next, the descriptions were matched to the corresponding concept to include SNOMED CT concepts that were misspelled ('nonsmall cell lung cancer' in Fig. 4.2). Once the concepts are detected, the algorithm adds these as origin or

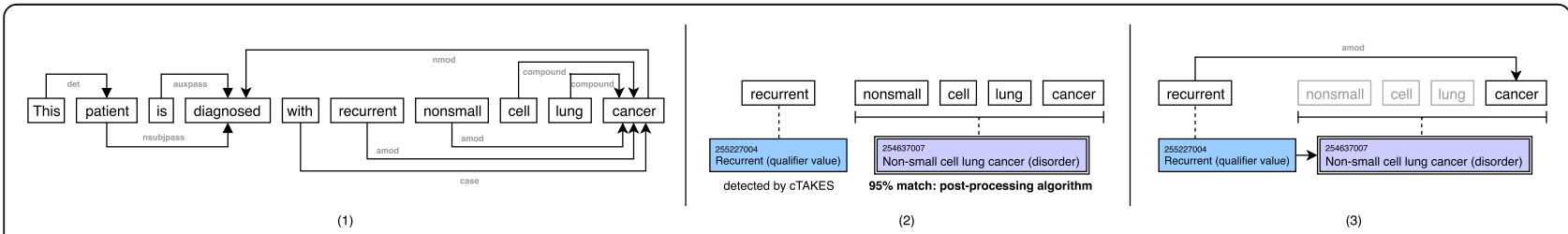

Fig. 4 Transformation of the syntactic relationships in the sentence 'This patient is diagnosed with recurrent nonsmall cell lung cancer' (1) to a SNOMED CT concept (2) and a relationship between detected SNOMED CT concepts (3, Recurrent and Non-small cell lung cancer) 
destination in the relationship (relationship between 'recurrent' and 'Non-small cell lung cancer' in Fig. 4.3).

Attribute relationship detection The algorithm to detect attribute relationships analyzes all relationships and searches a possible attribute relationship between the two concepts by using the SNOMED CT Machine Readable Concept Model (MRCM). The MRCM represents the SNOMED CT concept model rules and it includes domains (e.g. Clinical finding), attributes (e.g. Clinical course), and ranges (e.g. Courses) [23]. The attribute relationship detection algorithm retrieves all possible attribute type SNOMED CT concepts (predicates) that link the destination concept (object) to the source concept (subject) and it adds this attribute concept to the relationship to form a new post-coordinated expression (e.g. Non-small cell lung cancer with Recurrent as Clinical course, Fig. 4).

Custom dictionary Version 2016AB of the UMLS was converted to a cTAKES dictionary using the cTAKES dictionary creator [24]. A custom, plain-text, cTAKES dictionary was developed to link specific keywords that are not included in the UMLS to UMLS concepts. These UMLS concepts are converted to the corresponding SNOMED CT concept in the processing of cTAKES' output. Words that can be spelled in different ways, such as 'recurrence' will now also map to the right concept, such as '255227004 | Recurrent (qualifier value) |'.

\section{Evaluation of the developed MLP tool}

To determine which aspect of DIRECT adds value to the annotation of free text, we compared DIRECT to different implementations: cTAKES with the 2011 UMLS version (out-of-the-box, UMLS2011), cTAKES with the 2016AB UMLS version (UMLS2016), and cTAKES with the 2016AB UMLS version and a custom dictionary (UMLS2016 + Dict.). The free-text clinical notes were used as input for each implementation. The output for named-entity recognition and attribute relationship detection was collected and compared to the manual annotation using an $\mathrm{R}$ script. Named-entity recognition focused on the detection of SNOMED CT concepts 93880001 | Primary malignant neoplasm of lung (disorder) |, 254637007 | Non-small cell lung cancer (disorder) |, and 255227004 | Recurrent (qualifier value) |. Attribute relationship detection focused on the detection of the SNOMED CT relationship 93880001 | Primary malignant neoplasm of lung (disorder) |: 263502005 | Clinical course (attribute) |=255227004 | Recurrent (qualifier value) |. This resulted in the number of true positives, false positives, true negatives, and false negatives. These classifications were then used to calculate the precision, recall, and the $F_{1}$-score (Eq. 1, Eq. 2 and Eq. 3, Table 1), the harmonic mean of precision and recall. To compare the performance of the four implementations, a twotailed permutation test was used, since our dataset is small [25].

$$
\begin{aligned}
& \text { Precision }=\frac{T P}{T P+F P} \\
& \text { Recall }=\frac{T P}{T P+F N} \\
& \text { F1-score }=2 \cdot \frac{\text { Precision } \cdot \text { Recall }}{\text { Precision }+ \text { Recall }}
\end{aligned}
$$

\section{Used charts}

For the evaluation of DIRECT, 98 English (non-small cell) lung cancer patient charts in the form of treatment progress notes from six different centers were included (Table 2). The cases were provided and approved for use by Jonn Wu (JW), an oncologist at BC Cancer, Canada, and manually annotated by a student (Shan Rajapakshe). Both JW and MK determined if the annotations were of good quality. Thirty randomly selected charts are used as development set. The charts are assigned a positive, negative, or not-

Table 1 Variables used in the F-score equation

\begin{tabular}{llll}
\hline Algorithm & True positive (TP) & False positive (FP) & False negative (FN) \\
\hline Named-entity recognition $^{\text {a }}$ & $\begin{array}{l}\text { Same medical concept identified as } \\
\text { golden standard. }\end{array}$ & $\begin{array}{l}\text { Identified medical concept differs from } \\
\text { golden standard. }\end{array}$ & $\begin{array}{l}\text { Medical concept mentioned, } \\
\text { but not identified. }\end{array}$ \\
Attribute relationship detection ${ }^{\text {b }}$ & $\begin{array}{l}\text { Attribute relationship present and } \\
\text { detected. }\end{array}$ & $\begin{array}{l}\text { Attribute relationship not present, but } \\
\text { detected. }\end{array}$ & $\begin{array}{l}\text { Attribute relationship present, } \\
\text { but not detected. }\end{array}$ \\
\hline
\end{tabular}

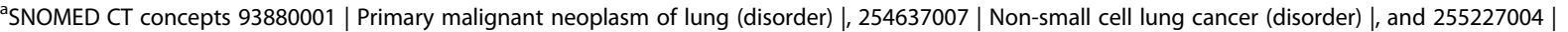
Recurrent (qualifier value)

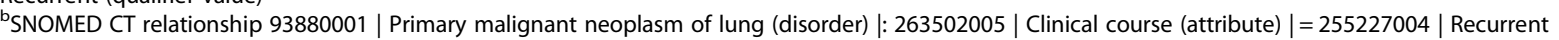
(qualifier value)

'SNOMED CT concept 255227004 | Recurrent (qualifier value) 
Table 2 Specification of the included charts

\begin{tabular}{|c|c|c|c|c|c|c|c|}
\hline \multirow[t]{2}{*}{ Set } & \multirow[t]{2}{*}{ Outcome } & \multicolumn{2}{|c|}{ Lung cancer } & \multicolumn{2}{|c|}{ Non-small cell lung cancer } & \multirow{2}{*}{$\begin{array}{l}\text { Recurrence } \\
\text { Implied }\end{array}$} & \multirow{2}{*}{$\begin{array}{l}\text { Relatior } \\
\text { Strict }\end{array}$} \\
\hline & & Implied & Strict & Implied & Strict & & \\
\hline \multirow[t]{3}{*}{ Development set $(n=30)$} & Positive & 27 & 23 & 17 & 17 & 10 & 6 \\
\hline & Negative & - & - & - & - & 5 & - \\
\hline & Not listed & 3 & 7 & 13 & 13 & 15 & 13 \\
\hline \multirow[t]{3}{*}{ Test set $(n=68)$} & Positive & 51 & 40 & 36 & 31 & 20 & 10 \\
\hline & Negative & - & - & - & - & 10 & - \\
\hline & Not listed & 17 & 28 & 32 & 37 & 38 & 58 \\
\hline
\end{tabular}

Relation: Relationship between Lung cancer and Recurrence

listed label, based on the occurrence (independent of the number of concept mentions) of the concepts. This label will be leveraged as reference standard in the evaluation process. $50 \%$ of the development set and $44 \%$ of the test set consist of charts that mention recurrence, either positive or negative.

Named-entity recognition is evaluated with an implied and a strict approach for the detection of lung cancer and non-small cell lung cancer. The implied approach includes charts that imply that there is an instance of the concept, but do not name the concept, e.g. 'nonsmall cell' instead of 'non-small cell lung cancer'. The strict approach only focuses on the charts that do name the concept.

\section{Results}

\section{Development of DIRECT}

The concepts were added to the dictionary, according to the methods described. The resulting custom dictionary can be found in Appendix A. DIRECT and the cTAKES API were developed over a period of 4 months by one developer (MK). Figure 5 shows the workflow in the application. Users enter text from a clinical source (Fig. 5.1) and select specific concepts (e.g. Primary malignant neoplasm of lung, Fig. 5.2) and their children, or the relationship between concepts (e.g. Small cell carcinoma of lung with Recurrent as Clinical course, Fig. 5.3) to focus on. The results of the annotation of the free text are then shown to the user (Fig. 5.4).

\section{Evaluation of the algorithms of DIRECT}

Table 3 shows the outcomes of the evaluation of named-entity recognition and attribute relationship detection for the three concepts, the data used for calculating the metrics (true positives, false positives, and false negatives) can be found in Appendix $B$. The calculated $F_{1}$-scores can also be found in Table 4 . The results are shown resp. for the implementations, the implied and strict approach, and the actual presence and absence of the concept in the text. All relationships in the development set were detected, however, not all relationships in the test set were detected. In all but one of the cases, the $F_{1}$-score of DIRECT was higher than the scores of the other implementations, the only exception being non-small cell lung cancer, having an $\mathrm{F}_{1}$-score that was higher in the UMLS2011 implementation. The UMLS2016 + Dict. implementation and DIRECT did detect 'recurrence', where the UMLS2011 and UMLS2016 implementations did not. DIRECT did not detect recurrence in one case.

The results of the two-tailed permutation test for comparison between the different implementations and DIRECT are shown in Table 5. The test showed statistically significant differences $(P<0.05)$ in outcomes between DIRECT and the UMLS2016 implementation for the detection of lung cancer and non-small cell lung, demonstrating that these differences are not coincidental [25]. There were no significant differences between the detection of lung cancer and non-small cell lung cancer in the original cTAKES (with UMLS 2011) and DIRECT, despite the higher Fscores for the detection of lung cancer. However, there was a significant difference between the detection of recurrence in these implementations.

\section{Discussion}

In this study we implemented DIRECT, a custom version of cTAKES that includes an API and MLP algorithms, and evaluated it using (non-small cell) lung cancer cases. Our results show that DIRECT can identify lung cancer, non-small cell lung cancer and recurrence concepts in charts with precisions of 1.000, 0.966, and 0.879 , either with implied and strict approaches, compared to strict precisions of $0.943,0.967$, and 0.000 in the original cTAKES implementation.

Use of either the UMLS2011 or UMLS2016 dictionary results in statistically significant differences in $\mathrm{F}_{1}$-scores for named-entity recognition. The old 


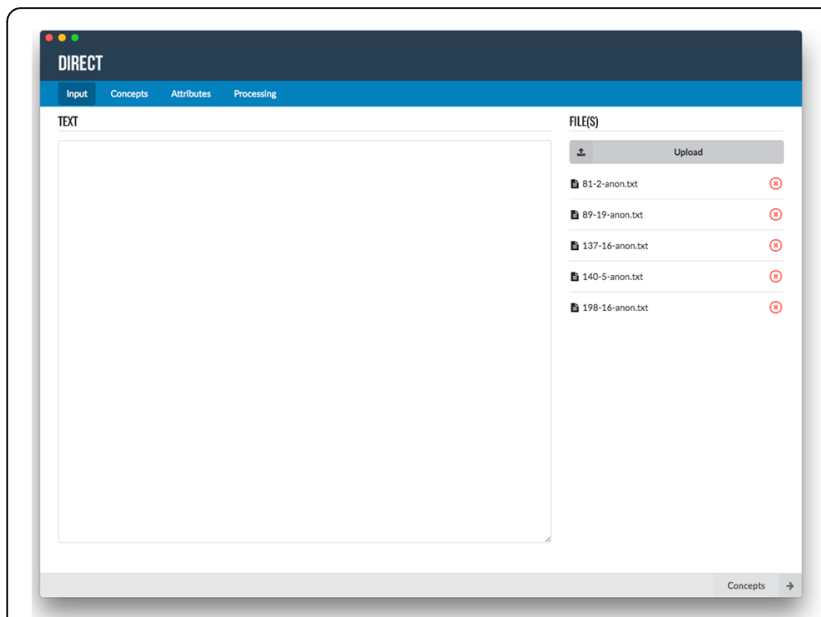

(1)

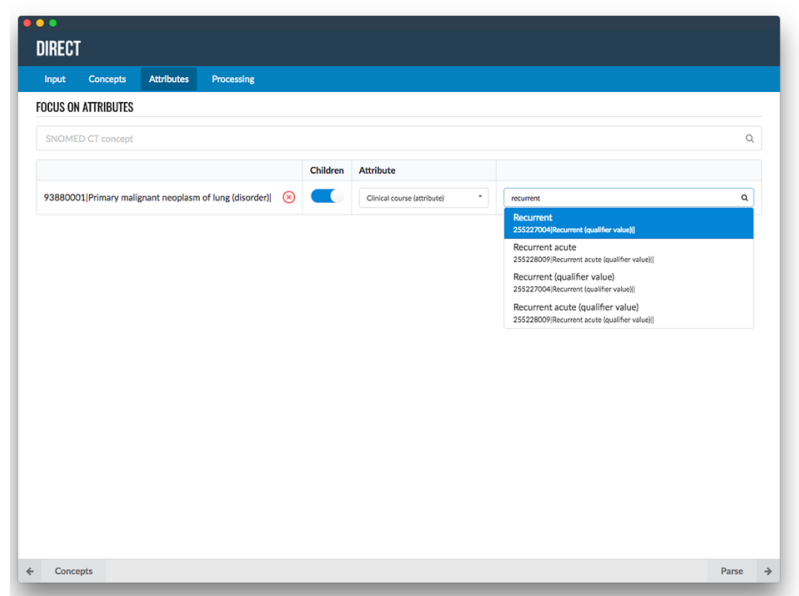

(3)

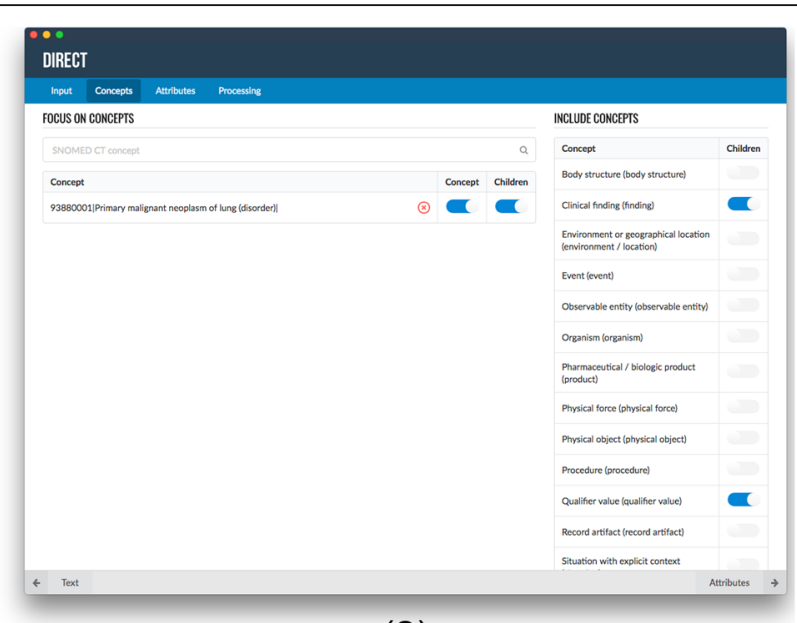

(2)

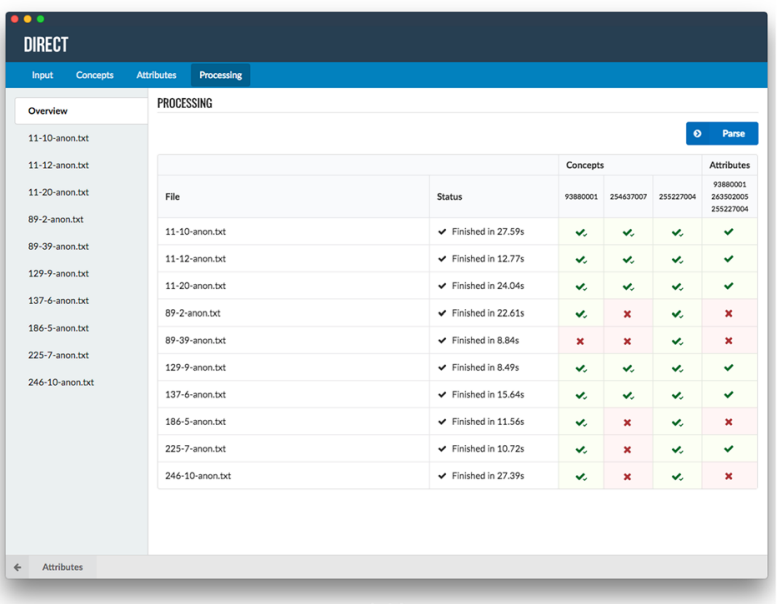

(4)

Fig. 5 Screenshots of DIRECT. 1. Input free text using a text field or text file(s). 2. Selection of SNOMED CT concepts to focus on and top-level concepts to include. 3. Selection of SNOMED CT attribute relationships to focus on. 4. Processing of the free text and results

dictionary (2011) consists of all the synonyms of SNOMED CT, NCI Thesaurus, MeSH, and ICD-9 whereas the new dictionary (2016) solely consists of SNOMED CT concepts and descriptions. Therefore, many concepts in the new dictionary have less descriptions and are thus harder to detect using the algorithm. DIRECT does detect these concepts with its algorithms, however with an $\mathrm{F}_{1}$-score for the detection of lung cancer that does not differ significantly from that of the UMLS2011 implementation.

The statistically significant differences in $F_{1}$-scores between the UMLS2011 implementation and DIRECT for non-small cell lung cancer can be explained by the relationship detection algorithm. The noun phrase as adverbial modifier relationship (npadvmod) was not included in the relationship detection. One case that contains this relationship between non-small cell and lung cancer could not be detected due to the missing relationship in the algorithm.

One chart included the text 'recurrent disease'. Since this description is longer than 'recurrent', DIRECT chose the SNOMED concept for recurrent disease over the concept for recurrent. This explains why the $\mathrm{F}_{1}$-score for the detection of recurrence is higher in the UMLS2016 + Dict. implementation compared to DIRECT.

The relationship 'lung cancer with recurrent as clinical course' is detected with an $\mathrm{F}_{1}$-score of 0.857 . cTAKES does detect relationships between complex adjective and noun combinations (e.g. 'Recurrent T3 NO nonsmall cell (adenocarcinoma) lung cancer' or 'recurrence of stage IIB non-small lung cancer' instead of 'recurrent non-small cell lung cancer'), however it labels them with the wrong syntactic category. 
Table 3 Precision, recall and calculated F-scores from the evaluation outcomes

\begin{tabular}{|c|c|c|c|c|c|c|c|c|c|}
\hline \multirow[b]{2}{*}{ Implementation } & \multirow[b]{2}{*}{ Algorithm } & \multirow[b]{2}{*}{ Concept } & \multirow[b]{2}{*}{ Approach } & \multicolumn{3}{|c|}{$\begin{array}{l}\text { Development set } \\
(n=30)\end{array}$} & \multicolumn{3}{|c|}{ Test set $(n=68)$} \\
\hline & & & & Precision & Recall & F-score & Precision & Recall & F-score \\
\hline$\overline{\text { UMLS2011 }}$ & \multirow{5}{*}{$\begin{array}{l}\text { Named-entity } \\
\text { recognition }\end{array}$} & \multirow[t]{2}{*}{ Lung cancer $^{a}$} & Implied & 0.938 & 0.556 & 0.698 & 1.000 & 0.686 & 0.814 \\
\hline \multirow[t]{4}{*}{ cTAKES with UMLS 2011} & & & Strict & 0.938 & 0.652 & 0.769 & 0.943 & 0.825 & 0.880 \\
\hline & & \multirow{2}{*}{$\begin{array}{l}\text { Non-small cell } \\
\text { lung cancer }\end{array}$} & Implied & 0.917 & 0.647 & 0.759 & 0.967 & 0.806 & 0.879 \\
\hline & & & Strict & 0.917 & 0.647 & 0.759 & 0.967 & 0.935 & 0.951 \\
\hline & & Recurrence $^{c}$ & & 0.000 & 0.000 & 0.000 & 0.000 & 0.000 & 0.000 \\
\hline UMLS2016 & \multirow{5}{*}{$\begin{array}{l}\text { Named-entity } \\
\text { recognition }\end{array}$} & \multirow[t]{2}{*}{ Lung cancer ${ }^{\mathrm{a}}$} & Implied & 1.000 & 0.370 & 0.541 & 1.000 & 0.569 & 0.725 \\
\hline \multirow[t]{4}{*}{ cTAKES with UMLS 2016} & & & Strict & 1.000 & 0.435 & 0.606 & 1.000 & 0.725 & 0.841 \\
\hline & & \multirow{2}{*}{$\begin{array}{l}\text { Non-small cell } \\
\text { lung cancer }\end{array}$} & Implied & 1.000 & 0.294 & 0.455 & 0.947 & 0.500 & 0.655 \\
\hline & & & Strict & 1.000 & 0.294 & 0.455 & 0.947 & 0.581 & 0.720 \\
\hline & & Recurrence $^{c}$ & & 0.000 & 0.000 & 0.000 & 0.000 & 0.000 & 0.000 \\
\hline UMLS2016 + Dict. & \multirow{5}{*}{$\begin{array}{l}\text { Named-entity } \\
\text { recognition }\end{array}$} & \multirow[t]{2}{*}{ Lung cancer ${ }^{a}$} & Implied & 1.000 & 0.852 & 0.920 & 1.000 & 0.706 & 0.828 \\
\hline \multirow{4}{*}{$\begin{array}{l}\text { CTAKES with UMLS } 2016 \text { and custom } \\
\text { dictionary }\end{array}$} & & & Strict & 1.000 & 1.000 & 1.000 & 1.000 & 0.900 & 0.947 \\
\hline & & \multirow{2}{*}{$\begin{array}{l}\text { Non-small cell } \\
\text { lung cancer }\end{array}$} & Implied & 1.000 & 0.765 & 0.867 & 0.957 & 0.611 & 0.746 \\
\hline & & & Strict & 1.000 & 0.765 & 0.867 & 0.957 & 0.710 & 0.815 \\
\hline & & Recurrence $^{c}$ & & 1.000 & 1.000 & 1.000 & 0.882 & 1.000 & 0.938 \\
\hline DIRECT & \multirow{5}{*}{$\begin{array}{l}\text { Named-entity } \\
\text { recognition }\end{array}$} & \multirow[t]{2}{*}{ Lung cancer $^{\mathrm{a}}$} & Implied & 1.000 & 0.852 & 0.920 & 1.000 & 0.706 & 0.828 \\
\hline \multirow{5}{*}{$\begin{array}{l}\text { CTAKES with UMLS 2016, custom dictionary, } \\
\text { and additional processing }\end{array}$} & & & Strict & 1.000 & 1.000 & 1.000 & 1.000 & 0.900 & 0.947 \\
\hline & & \multirow{2}{*}{$\begin{array}{l}\text { Non-small cell } \\
\text { lung cancer }\end{array}$} & Implied & 1.000 & 1.000 & 1.000 & 0.966 & 0.778 & 0.862 \\
\hline & & & Strict & 1.000 & 1.000 & 1.000 & 0.966 & 0.903 & 0.933 \\
\hline & & Recurrence $^{c}$ & & 1.000 & 1.000 & 1.000 & 0.879 & 0.967 & 0.921 \\
\hline & $\begin{array}{l}\text { Attribute relationship } \\
\text { detection }\end{array}$ & $\begin{array}{l}\text { Recurrent lung } \\
\text { cancer }^{d}\end{array}$ & & 1.000 & 1.000 & 1.000 & 1.000 & 0.750 & 0.857 \\
\hline
\end{tabular}

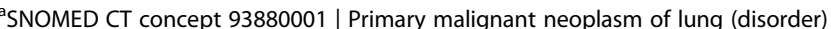

bSNOMED CT concept 254637007 | Non-small cell lung cancer (disorder)

'SNOMED CT concept 255227004 | Recurrent (qualifier value)

dRelationship between three SNOMED CT concepts: 93880001 | Primary malignant neoplasm of lung (disorder) |: 263502005 | Clinical course (attribute) |= 255227004 | Recurrent (qualifier value)

Table 4 F-scores calculated from the evaluation outcomes

\begin{tabular}{|c|c|c|c|c|c|c|c|c|c|c|}
\hline \multirow[b]{2}{*}{ Algorithm } & \multirow[b]{2}{*}{ Concept } & \multirow[b]{2}{*}{ Approach } & \multicolumn{4}{|c|}{ Development set $(n=30)$} & \multicolumn{4}{|c|}{ Test set $(n=68)$} \\
\hline & & & UMLS2011 & UMLS2016 & UMLS2016D & $\overline{\text { DIRECT }}$ & UMLS2011 & UMLS2016 & UMLS2016D & DIRECT \\
\hline \multirow[t]{5}{*}{ Named-entity recognition } & \multirow[t]{2}{*}{ Lung cancer ${ }^{a}$} & Implied & 0.698 & 0.541 & 0.920 & 0.920 & 0.814 & 0.725 & 0.828 & 0.828 \\
\hline & & Strict & 0.769 & 0.606 & 1.000 & 1.000 & 0.880 & 0.841 & 0.947 & 0.947 \\
\hline & \multirow{2}{*}{$\begin{array}{l}\text { Non-small cell } \\
\text { lung cancer }\end{array}$} & Implied & 0.759 & 0.455 & 0.867 & 1.000 & 0.879 & 0.655 & 0.746 & 0.862 \\
\hline & & Strict & 0.759 & 0.455 & 0.867 & 1.000 & 0.951 & 0.720 & 0.815 & 0.933 \\
\hline & Recurrence $^{c}$ & & 0.000 & 0.000 & 1.000 & 1.000 & 0.000 & 0.000 & 0.938 & 0.921 \\
\hline Relationship detection & $\begin{array}{l}\text { Recurrent lung } \\
\text { cancer }^{d}\end{array}$ & & & & & 1.000 & & & & 0.857 \\
\hline
\end{tabular}

\footnotetext{
${ }^{a}$ SNOMED CT concept 93880001 | Primary malignant neoplasm of lung (disorder)
}

bSNOMED CT concept 254637007 | Non-small cell lung cancer (disorder)

'SNOMED CT concept 255227004 | Recurrent (qualifier value)

${ }^{\mathrm{d}}$ Relationship between three SNOMED CT concepts: 93880001 | Primary malignant neoplasm of lung (disorder) |: 263502005 | Clinical course (attribute) |= 255227004 | Recurrent (qualifier value) 
Table 5 Outcomes of the two-tailed permutation test between the different implementations Statistically significant values $(p<0.05)$ are in bold face

\begin{tabular}{|c|c|c|c|c|}
\hline Implementation 1 & Implementation 2 & Lung $_{\text {cancer }}{ }^{\mathrm{a}}$ & Non-small cell lung cancer ${ }^{b}$ & Recurrence \\
\hline UMLS2011 & UMLS2016 & 0.024 & 0.002 & 1.000 \\
\hline UMLS2011 & UMLS2016 + Dict. & 1.000 & 0.016 & $<0.001$ \\
\hline UMLS2011 & DIRECT & 1.000 & 1.000 & $<0.001$ \\
\hline UMLS2016 & UMLS2016 + Dict. & 0.024 & 0.142 & $<0.001$ \\
\hline UMLS2016 & DIRECT & 0.018 & 0.001 & $<0.001$ \\
\hline UMLS2016 + Dict. & DIRECT & 1.000 & 0.035 & 1.000 \\
\hline
\end{tabular}

${ }^{\mathrm{a}} \mathrm{SNOMED}$ CT concept 93880001 | Primary malignant neoplasm of lung (disorder)

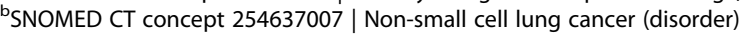

'SNOMED CT concept 255227004 | Recurrent (qualifier value)

Therefore, the algorithm cannot detect the attribute relationship. This problem might be solved in DIRECT by changing the algorithm to include other syntactic relationships as well.

The $\mathrm{F}_{1}$-scores show that DIRECT can substantially reduce manual chart abstraction efforts for these concepts. Possible reasons for not tagging concepts are spelling mistakes that are off by more than $5 \%$ from the SNOMED CT concept description or concept relationships in texts that are not detected, such as charts that mention cancer in one sentence and specify it as non-small cell in another sentence.

Strengths of our study include the customizability of the dictionaries and the selection of focus concepts in the user interface. This makes DIRECT generic (i.e. noninstitution-specific) and allows it to be used for different study designs. Moreover, the algorithms used in DIRECT are generic and therefore not bound to non-small cell lung cancer charts. The algorithms are described in detail, thus these algorithms can be replicated in other implementations. We also used a UMLS dictionary, which is scalable due to the large quantity and variety of concepts available in the UMLS.

Several limitations of our study should be noted. The number of cases is acceptable, but a larger dataset could be used to give the evaluation more power. Additionally, the split-sample evaluation exclusively focussed on (non-small cell) lung cancer with cases that had limited (e.g. 'nonsmall cell') to no misspelled concepts and is not externally validated. We did not use cross-validation, since the creation of custom dictionaries and the development of the algorithms based on the development data is highly labour-intensive. Other implementations or cases could give other outcomes, partly due to the non-scalable non-small cell lung cancer custom dictionary.

Comparing the outcome of the algorithms with algorithms found in literature is challenging, since algorithms are often developed for specific implementations and evaluated by encoding specific free-text narratives. We therefore could not compare our outcomes to outcomes described in other MLP papers.

Complete and structured EHR data can improve health care by allowing data to be reused for research and development of clinical decision support tools [4]. This study found that DIRECT can be used to detect specific oncology concepts in free text. We believe that DIRECT and the algorithms described in this paper may be used in other medical settings as well. Clinicians can use DIRECT to get acquainted with MLP, without building their own MLP pipelines. Batches of free text can be processed by the CTAKES API, saving time for clinicians and researchers, who would otherwise have to abstract information manually.

Future studies should validate MLP tools such as cTAKES and DIRECT using cross-validation and external validation and should investigate a different implementation for (non-small cell) lung cancer or other specialisms. Furthermore, the performance of the relationship detection algorithm could be improved and further research is needed to provide methods for that. Future studies should also critically assess the encoded attribute relationships, since DIRECT does not determine if the detected relationships are clinically correct and relevant.

\section{Conclusion}

In this study we developed and evaluated the MLP tool DIRECT, an implementation of cTAKES. We demonstrated how DIRECT could be used to detect oncology concepts through a web interface and how it could detect attribute relationships using MLP algorithms with significant increase in $\mathrm{F}_{1}$-score, compared to the original implementation of cTAKES. DIRECT can be used to encode clinical narratives, and thus substantially reduce manual chart abstraction efforts, saving time for clinicians and researchers. 


\section{Appendix}

Table 6 Contents of the custom dictionary

\begin{tabular}{|c|c|c|c|c|}
\hline$\overline{\mathrm{CUI}}$ & Concept description & Type & Type description & Words \\
\hline C2945760 & Recurrent & T079 & Temporal Concept & recurrent \\
\hline C2945760 & Recurrent & T079 & Temporal Concept & recurring \\
\hline C2945760 & Recurrent & T079 & Temporal Concept & recurrence \\
\hline C0007131 & Non-Small Cell Lung Carcinoma & T191 & Neoplastic Process & non-small cell lung carcinoma \\
\hline C0007131 & Non-Small Cell Lung Carcinoma & T191 & Neoplastic Process & nonsmall cell lung carcinoma \\
\hline C0007131 & Non-Small Cell Lung Carcinoma & T191 & Neoplastic Process & non-small cell lung adenocarcinoma \\
\hline C0007131 & Non-Small Cell Lung Carcinoma & T191 & Neoplastic Process & nonsmall cell lung adenocarcinoma \\
\hline C0007131 & Non-Small Cell Lung Carcinoma & T191 & Neoplastic Process & non-small cell carcinoma of the lung \\
\hline C0007131 & Non-Small Cell Lung Carcinoma & T191 & Neoplastic Process & nonsmall cell carcinoma of the lung \\
\hline C0007131 & Non-Small Cell Lung Carcinoma & T191 & Neoplastic Process & non-small cell adenocarcinoma of the lung \\
\hline C0007131 & Non-Small Cell Lung Carcinoma & T191 & Neoplastic Process & nonsmall cell adenocarcinoma of the lung \\
\hline C0007131 & Non-Small Cell Lung Carcinoma & T191 & Neoplastic Process & non small cell lung carcinoma \\
\hline C0007131 & Non-Small Cell Lung Carcinoma & T191 & Neoplastic Process & non small cell lung adenocarcinoma \\
\hline C0007131 & Non-Small Cell Lung Carcinoma & T191 & Neoplastic Process & non small cell carcinoma of the lung \\
\hline C0007131 & Non-Small Cell Lung Carcinoma & T191 & Neoplastic Process & non small cell adenocarcinoma of the lung \\
\hline C0149925 & Small cell carcinoma of lung & T191 & Neoplastic Process & small cell lung carcinoma \\
\hline C0149925 & Small cell carcinoma of lung & T191 & Neoplastic Process & small cell lung adenocarcinoma \\
\hline C0149925 & Small cell carcinoma of lung & T191 & Neoplastic Process & small cell carcinoma of the lung \\
\hline C0149925 & Small cell carcinoma of lung & T191 & Neoplastic Process & small cell adenocarcinoma of the lung \\
\hline C1306460 & Primary malignant neoplasm of lung & T191 & Neoplastic Process & lung carcinoma \\
\hline C1306460 & Primary malignant neoplasm of lung & T191 & Neoplastic Process & lung adenocarcinoma \\
\hline C1306460 & Primary malignant neoplasm of lung & T191 & Neoplastic Process & carcinoma of the lung \\
\hline C1306460 & Primary malignant neoplasm of lung & T191 & Neoplastic Process & adenocarcinoma of the lung \\
\hline
\end{tabular}

CUI Unified Medical Language System's Concept Unique Identifier 


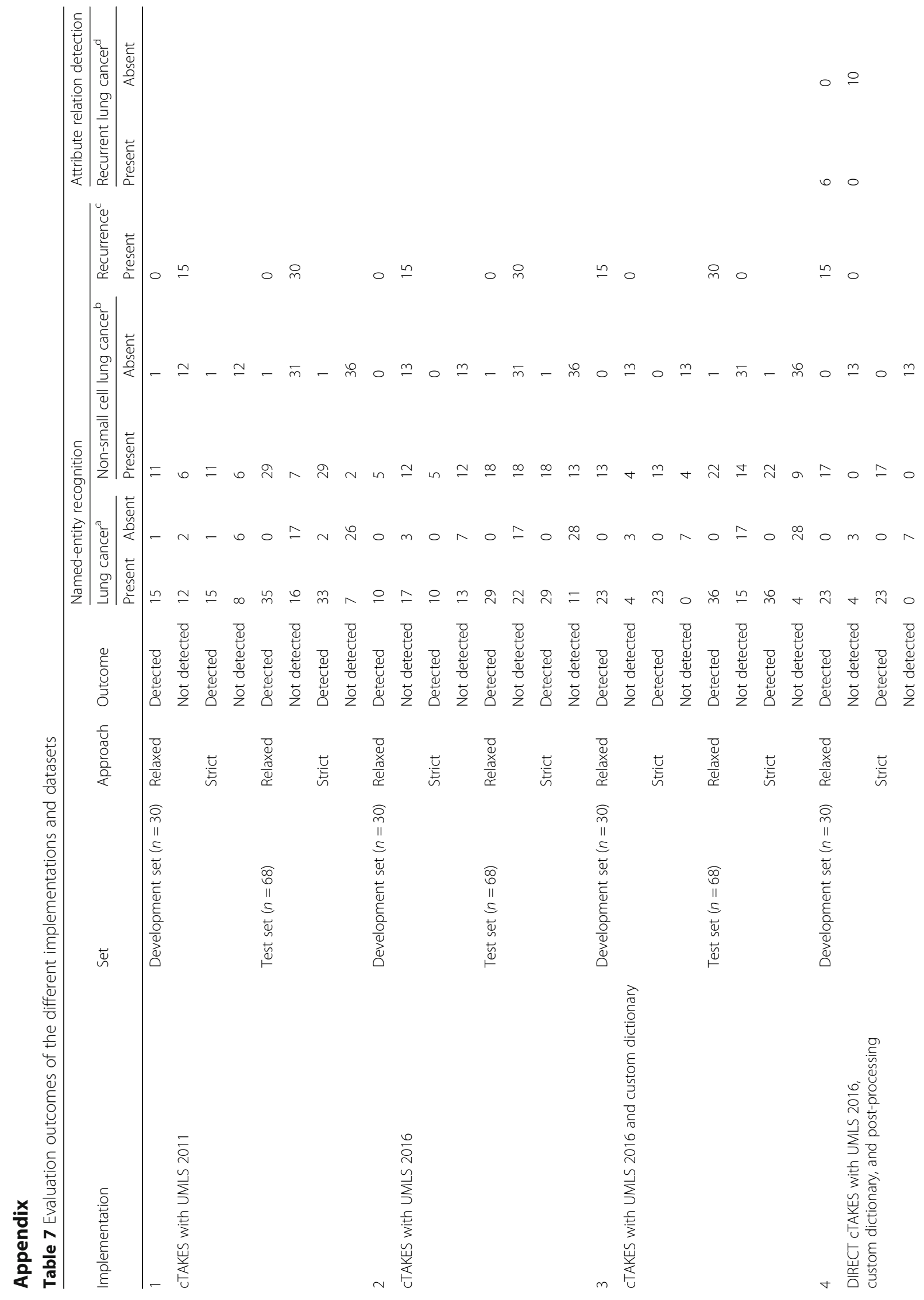




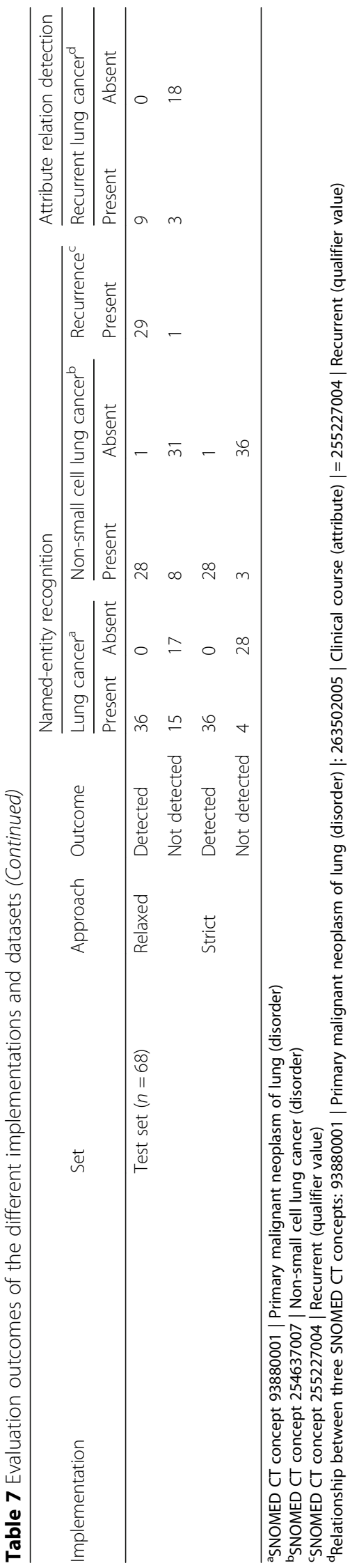




\section{Abbreviations}

API: Application Programming Interface; cTAKES: Clinical Text Analysis and Knowledge Extraction System; DIRECT: Disease Information and Relationship ExtraCtion Tool; EHR: Electronic Health Record; MLP: Medical Language Processing; MRCM: SNOMED CT Machine Readable Concept Model; NLP: Natural Language Processing; UIMA: Unstructured Information Management Architecture; UMLS: Unified Medical Language System

\section{Acknowledgements}

We would like to thank Jonn Wu and Shan Rajapakshe for providing and annotating the free-text patient charts. We would also like to thank Dennis Lee and Dennis Wellborn for technical assistance.

\section{Authors' contributions}

FL, RC, and MK designed and directed the project. AA and DA provided input on the methodology used in the project. MK developed and evaluated the MLP tool and drafted the manuscript. All authors read and approved the final manuscript.

\section{Funding}

This work was supported by RKL Health Informatics Ltd., Castor EDC, and the European Regional Development Fund (ERDF).

\section{Availability of data and materials}

The datasets used in the current study are not publicly available, for the researchers were granted access to the data for this study only. The source code of DIRECT can be found at https://github.com/martijnkersloot/direct and the R script used for the permutation test can be found at https://doi. org/10.6084/m9.figshare.7539494.

\section{Ethics approval and consent to participate}

Not applicable.

\section{Consent for publication}

Not applicable.

\section{Competing interests}

The authors declare that they have no competing interests.

\section{Author details}

${ }^{1}$ Department of Medical Informatics, Amsterdam Public Health Research Institute, Amsterdam UMC, University of Amsterdam, Meibergdreef 9, 1105AZ Amsterdam, The Netherlands. ${ }^{2}$ School of Health Information Science, University of Victoria, Victoria, Canada.

\section{Received: 18 January 2019 Accepted: 13 August 2019}

Published online: 18 September 2019

\section{References}

1. Meystre SM, Savova GK, Kipper-Schuler KC, Hurdle JF. Extracting information from textual documents in the electronic health record: a review of recent research. Yearb Med Inform. 2008:128-44. https://www.ncbi.nlm.nih.gov/ pubmed/18660887.

2. Zhou L, Mahoney LM, Shakurova A, Goss F, Chang FY, Bates DW, et al. How many medication orders are entered through free-text in EHRs?--a study on hypoglycemic agents. AMIA Annu Symp Proc AMIA Sym. 2012;2012:1079-88.

3. Ford E, Nicholson A, Koeling R, Tate A, Carroll J, Axelrod L, et al. Optimising the use of electronic health records to estimate the incidence of rheumatoid arthritis in primary care: what information is hidden in free text? BMC Med Res Methodol. 2013;13:105.

4. Wells BJ, Chagin KM, Nowacki AS, Kattan MW. Strategies for handling missing data in electronic health record derived data. EGEMS (Washington, DC). 2013;1(3):1035.

5. Liu H, Wu ST, Li D, Jonnalagadda S, Sohn S, Wagholikar K, et al. Towards a semantic lexicon for clinical natural language processing. AMIA Ann Symp Proc AMIA Symp. 2012;2012:568-76.

6. Szlosek DA, Ferrett J. Using machine learning and natural language processing algorithms to Automate the evaluation of clinical decision support in electronic medical record systems. EGEMS (Washington, DC). 2016;4(3):1222
7. Ruch P, Baud R, Geissbuhler A. Using lexical disambiguation and namedentity recognition to improve spelling correction in the electronic patient record. Artif Intell Med. 2003;29(1-2):169-84.

8. Salmasian H, Freedberg DE, Friedman C. Deriving comorbidities from medical records using natural language processing. J Am Med Inform Assoc. 2013;20(e2):e239-42.

9. Li Q, Melton K, Lingren T, Kirkendall ES, Hall E, Zhai H, et al. Phenotyping for patient safety: algorithm development for electronic health record based automated adverse event and medical error detection in neonatal intensive care 2014; 2014. p. 776-84.

10. Ni Y, Wright J, Perentesis J, Lingren T, Deleger L, Kaiser M, et al. Increasing the efficiency of trial-patient matching: automated clinical trial eligibility pre-screening for pediatric oncology patients. BMC Med Inform Decis Mak. 2015;15:28.

11. Carrell DS, Halgrim S, Tran DT, Buist DS, Chubak J, Chapman WW, et al. Using natural language processing to improve efficiency of manual chart abstraction in research: the case of breast cancer recurrence. Am J Epidemiol. 2014;179(6):749-58.

12. Zheng $L$, Wang $Y$, Hao $S$, Shin $A Y$, Jin $B$, Ngo AD, et al. Web-based real-time case finding for the population health Management of Patients with Diabetes Mellitus: a prospective validation of the natural language processing-based algorithm with statewide electronic medical records. JMIR Med Inform. 2016;4(4):e37.

13. Savova GK, Masanz JJ, Ogren PV, Zheng J, Sohn S, Kipper-Schuler KC, et al. Mayo clinical text analysis and knowledge extraction system (CTAKES): architecture, component evaluation and applications. J Am Med Inform Assoc. 2010;17(5):507-13.

14. SNOMED International. SNOMED CT [December 20, 2017]. Available from: https://www.snomed.org/snomed-ct.

15. U.S. National Library of Medicine. RxNorm 2014 [Available from: https:// www.nlm.nih.gov/research/umls/rxnorm/.

16. Masanz J, Pakhomov SV, Xu H, Wu ST, Chute CG, Liu H. Open source clinical NLP - more than any single system. AMIA Jt Summits Transl Sci Proc. 2014; 2014:76-82.

17. Kreimeyer K, Foster M, Pandey A, Arya N, Halford G, Jones SF, et al. Natural language processing systems for capturing and standardizing unstructured clinical information: a systematic review. J Biomed Inform. 2017;73:14-29.

18. Nadkarni PM, Ohno-Machado L, Chapman WW. Natural language processing: an introduction. J Am Med Inform Assoc. 2011;18(5):544-51.

19. National Library of Medicine (US). Introduction to the UMLS 2009 [December 19, 2017]. Available from: https:/www.ncbi.nlm.nih.gov/books/NBK9675/.

20. Choi JD, Palmer M. Guidelines for the clear style constituent to dependency conversion. Technical report 01-12; 2012.

21. Oliver I, Oliver I. Programming classics: implementing the world's best algorithms: Prentice Hall; 1993.

22. PHP Group. similar_text [October 31, 2018]. Available from: http://php.net manual/en/function.similar-text.php.

23. SNOMED International. SNOMED CT Machine Readable Concept Model 2017. Available from: https://confluence.ihtsdotools.org/display/DOCMRCM/ SNOMED+CT+Machine+Readable+Concept+Model. [March 29, 2017].

24. Finan S. Dictionary Creator GUI 2017. Available from: https:/cwiki.apache.org/ confluence/display/CTAKES/Dictionary+Creator+GUI. [December 19, 2017].

25. Dror R, Baumer G, Shlomov S, Reichart R, editors. The hitchhiker's guide to testing statistical significance in natural language processing. Proceedings of the 56th annual meeting of the Association for Computational Linguistics (Volume 1: Long Papers); 2018

\section{Publisher's Note}

Springer Nature remains neutral with regard to jurisdictional claims in published maps and institutional affiliations. 